\title{
Technical Feasibility and Oncological Legitimacy of Enucleation of Intraductal Papillary Mucinous Neoplasm Located at the Pancreatic Head or Uncinate Process
}

\author{
YUJI SOEJIMA ${ }^{1,2}$, TAKEO TOSHIMA ${ }^{2}$, TAKASHI MOTOMURA ${ }^{2}$, TOMOYUKI YOKOTA ${ }^{3}$, KOUJI JOKO ${ }^{3}$, \\ YUMI OSHIRO $^{4}$, IKUO TAKAHASHI ${ }^{2}$, TAKASHI NISHIZAKI ${ }^{2}$ and YOSHIHIKO MAEHARA ${ }^{1}$ \\ ${ }^{1}$ Departments of Surgery and Science, Graduate School of Medical Sciences, Kyushu University, Fukuoka, Japan; \\ Departments of ${ }^{2}$ Surgery, ${ }^{3}$ Hepatobiliary and Pancreatic Medicine, and \\ ${ }^{4}$ Pathology, Matsuyama Red Cross Hospital, Ehime, Japan
}

\begin{abstract}
Background: Surgical indications and strategies for branch duct (BD)-intraductal papillary mucinous neoplasm (IPMN) at the pancreatic head $(P H)$ are controversial issues. We investigated the technical feasibility and oncological legitimacy of enucleation for IPMN of the PH. Patients and Methods: The clinicopathological parameters of 11 patients with IPMN who underwent conventional pancreaticoduodenectomy $(P D, n=7)$ or enucleation $(n=4)$ during the same period were evaluated. Results: The mean operative time (442 vs. $280 \mathrm{~min}, p<0.05$ ) and blood loss (864 vs. $93 \mathrm{ml}, p<0.05)$ were significantly better in the enucleation group. The final pathological diagnosis (low-/intermediate-/high-grade dysplasia) in the $P D$ and enucleation groups was $4 / 2 / 1$ and $3 / 1 / 0$, respectively. The incidence of grade B pancreatic fistula and Clavien III complications was $14 \%$ vs. $0 \%$ and $43 \%$ vs. $25 \%$, respectively. No recurrent pancreatitis or exocrine or endocrine dysfunction was noted in the enucleation group; however, de novo or exacerbated diabetes mellitus developed in three patients in the PD group. All patients were alive and no disease recurrence was noted at a mean follow-up of 1,059 days. Conclusion: Enucleation of BD-IPMN at the PH is a technically feasible and oncologically acceptable procedure which should be justified in patients with IPMNs with a low risk of malignancy.
\end{abstract}

\footnotetext{
Correspondence to: Yoshihiko Maehara, MD, Ph.D, FACS and Yuji Soejima, MD, Ph.D., FACS, Departments of Surgery and Science, Graduate School of Medical Sciences, Kyushu University, Fukuoka 812-8582, Japan. Tel: +81 926425466, Fax: +81 926425482, e-mail: ysoejima@surg2.med.kyushu-u.ac.jp
}

Key Words: Intraductal papillary mucinous neoplasm, enucleation, pancreatic head, uncinate process, limited resection.
The use of modern abdominal imaging modalities such as computed tomography, magnetic resonance cholangiopancreatography, and endoscopic ultrasonography (EUS) has resulted in an increase in the incidental diagnosis of intraductal papillary mucinous neoplasm (IPMN) (1). Accordingly, surgical resection of IPMN has been recently increasing, which has accelerated the understanding of the clinicopathological features of this disease. However, the surgical indications, especially for branch duct (BD)-IPMN, remain uncertain. Previous studies have shown that the overall malignancy rate of resected BD-IPMN is only $25 \%$; this includes high-grade dysplasia (18\%) and invasive carcinoma (5\%) (2-4). Moreover, it is likely that the real incidence of malignancy of BD-IPMN is much lower because experienced clinicians are more likely to perform resection in patients at an increased risk of malignancy. These data suggest that the preoperative diagnosis of malignant BDIPMN and its operative indications remain very difficult. Nonetheless, irrespective of this low rate of malignancy, standard pancreatectomy such as pancreaticoduodenectomy (PD) is still indicated in most centers, largely because the technical feasibility and oncological legitimacy of less invasive techniques such as enucleation or uncinate process (UP) resection are unknown. The morbidity and mortality rates associated with PD are reportedly very high (as high as $50 \%$ and $5 \%$, respectively), even at high-volume centers (5). Furthermore, the incidence of long-term endocrine and exocrine insufficiency after PD is known to range from $20 \%$ to $50 \%$ and $40 \%$ to $70 \%$, respectively $(6,7)$. Therefore, balancing the risks and benefits of standard pancreatectomy for BD-IPMN located at the pancreas head (PH) and UP should be challenged and reconsidered. Enucleation, including that performed with laparoscopy, has recently been reported to be the treatment option for benign and low-grade malignant tumors such as neuroendocrine and other cystic tumors $(8,9)$. However, there are very few published series 
of enucleation for IPMN (10-12). Nonetheless, the oncological legitimacy of enucleation has not been evaluated yet. In the present study, we investigated the technical feasibility and oncological legitimacy of enucleation versus PD for IPMN located at the PH or UP.

\section{Patients and Methods}

Patients. From November 2011 to December 2015, a total of 11 consecutive patients with BD-IPMN located at the PH $(n=7)$ or UP $(n=4)$ underwent surgical resection at our institution. From a technical viewpoint, all patients were judged to be amenable to enucleation. The indications for surgery were possible malignancy $(n=8)$ and repeated pancreatitis $(n=3)$. In selected patients (Figure 1a and $b$ ), enucleation was indicated for IPMNs with a low risk of malignancy with $(n=3)$ or without $(n=1)$ a history of repeated pancreatitis (Table I).

Surgical procedures of enucleation. An endoscopic nasal pancreatic drainage (ENPD) tube was inserted several days before surgery for all patients undergoing enucleation. The surgical procedure for enucleation was performed as follows. Briefly, the abdomen was entered by an upper midline incision. Intraoperative ultrasound was then performed to identify and examine the nature of the tumors, especially the size and height of mural nodules, for which enucleation would be applicable. After exposing the infrapancreatic superior mesenteric vein, the UP was dissected off of the superior mesenteric vein and duodenum, while preserving the marginal inferior pancreaticoduodenal artery and veins (Figure 1c). Kocher's maneuver was not necessary in two cases. In most cases, the tumor was identifiable from the surface of the pancreas. The tumor was carefully dissected off of the pancreatic parenchyma with a mosquito dissector and electric cautery. The dissection was usually easy and clear; however, extreme care was taken to avoid injuring the tumor wall and inducing spillage of mucus. Small vessels were either ligated with a suture or divided with a sealing device such as the LigaSure ${ }^{\mathrm{TM}}$ Small Jaw Vessel-Sealing System (Covidien, Irvine, CA, USA). When dissection between the tumor and the pancreatic parenchyma was difficult, the side of the pancreatic parenchyma was ligated and divided while a small portion of the parenchyma was left on the cyst wall. Completion of the dissection always resulted in connection of the cyst to the main pancreatic duct only by its stalk (Figure 1d). After clamping the stalk with a bulldog clamp, intraoperative pancreatography was performed through the ENPD tube to confirm the integrity of the main pancreatic duct. The root of the stalk was carefully ligated with a silk suture and closed with continuous 6-0 PDS II (Ethicon, Inc., Somerville, NJ, USA) sutures. The resected stump was then divided and sent for fresh frozen pathological examination. Pancreatography was performed again through the ENPD tube to confirm the integrity of the main pancreatic duct. Surgery was finished when the pathological examination of the resected stump was negative for malignancy; both low- and intermediate-grade dysplasia was allowed. The amylase level of the drainage fluid was measured at 1,3, 5, and 7 days after surgery.

Surgical procedures of $P D$. The surgical procedures of $\mathrm{PD}$ are reported elsewhere (13). Briefly, after the completion of PD with conventional methods, invaginated pancreaticogastrostomy using an elastic suture was always performed.
Definition of pancreatic fistula and severity of complications. The definition of pancreatic fistula was that given by the International Study Group of Pancreatic Fistula (ISGPF) (14). The severity of postoperative complications was recorded according to the ClavienDindo classification (15).

Statistical analysis. Continuous variables were compared using the Mann-Whitney test for independent samples. All values are expressed as the mean \pm standard deviation. Categorical data were compared using the chi-square test. $p$-Values of less than 0.05 were considered statistically significant. All statistical analyses were performed using $\mathrm{JMP}^{\circledR}$ version 9.0.2. (SAS Institute, Inc., Cary, NC, USA).

\section{Results}

The intended procedures were completed in all patients. The patients' characteristics are summarized in Table I. Table II summarizes the clinicopathological outcomes. The mean tumor size in the PD and enucleation groups was 33 and $38 \mathrm{~mm}$, respectively, with no significant difference. The mean number of worrisome features was also comparable (1.7 in both groups). The mean operative time and blood loss volume were significantly better in the enucleation than the PD group ( $p<0.05$ for both). Only one patient in the enucleation group developed functional stenosis of the third portion of the duodenum that lasted for 60 days after surgery but healed with conservative treatment. The mean hospital stay in the PD and enucleation groups were 36 and 25 days, respectively (no significant difference). No recurrent pancreatitis, de novo diabetes mellitus, or exocrine insufficiency was noted in the enucleation group, while de novo $(\mathrm{n}=2)$ or exacerbated $(\mathrm{n}=1)$ diabetes mellitus developed in three patients in the PD group. All patients were alive at the time of this writing, and no disease recurrence was noted in either group at a mean followup of 1,059 days.

\section{Discussion}

The surgical indications for BD-IPMN have been an issue of debate because of the presumably low rate of malignancy of $26 \%$ (range $=6-47 \%$ ) in patients with surgically resected BDIPMNs as well as the low rate of progression. These rates are in sharp contrast to those of main duct IPMN, which has a malignancy rate of $60 \%$ (range $=11-81 \%$ ) (16). The revised 2012 International Consensus Guidelines for IPMN introduced two categories of risk factors for malignancy ("worrisome features" and "high-risk stigmata") to determine the treatment recommendations for BD-IPMN (16). IPMNs with high-risk stigmata should undergo surgical resection, while those with worrisome features, such as EUS, warrant further diagnostic evaluation and close follow-up. Observation is recommended for patients with asymptomatic cysts without any of these criteria. This guideline is now under worldwide validation; many authors have reported 
Table I. Characteristics of patients in this study.

\begin{tabular}{lccc}
\hline Factors & EN (n=4) & PD (n=7) & $p$-Value \\
\hline Age (years)* & $59 \pm 10$ & $74 \pm 4$ & $<0.05$ \\
Sex (M/F) (n) & $0 / 4$ & $2 / 5$ & NS \\
IPMN size (mm)* & $38 \pm 22$ & $33 \pm 16$ & NS \\
Location (uncus/head) (n) & $2 / 2$ & $3 / 7$ & NS \\
Multifocal IPMN (yes/no) (n) & $2 / 2$ & $3 / 4$ & NS \\
Mural nodule (yes/no) (n) & $2 / 2$ & $1 / 6$ & NS \\
MPD diameter (mm)* & $2.8 \pm 1.0$ & $2.3 \pm 1.1$ & NS \\
High-risk stigmata (yes/no) (n) & $0 / 4$ & $1 / 6$ & NS \\
No. of worrisome features & 1.7 & 1.7 & NS \\
History of pancreatitis (yes/no) (n) & $3 / 1$ & $2 / 5$ & NS \\
Cytology (n) & & & NS \\
Normal & 2 & 3 & \\
Atipical cells & 2 & 1 & \\
Malignant suspicious & 0 & 2 & \\
$\quad$ NA & 0 & 1 & \\
Tumor marker & & & \\
CEA (ng/ml)* & $1.7 \pm 0.8$ & $2.7 \pm 1.2$ & NS \\
CA19-9 (U/ml)* & $11.4 \pm 8.6$ & $23.7 \pm 20.3$ & NS \\
Indication for surgery (n) & & & NS \\
Repeated pancreatitis & 3 & 0 & \\
Possible malignancy & 1 & 7 & \\
\hline
\end{tabular}

*Mean \pm standard deviation. M: Males; F: females; IPMN: intraductal papillary mucinous neoplasm; EN: enucleation; PD: pancreaticoduodenectomy; MPD: main pancreatic duct; NA: not applicable; CEA: carcinoembryonic antigen; CA19-9, carbohydrate antigen 19-9; NS: not significant, $p \geq 0.05$.
Table II. Clinicopathological outcomes of patients in this study.

\begin{tabular}{lccc}
\hline Factors & EN (n=4) & PD (n=7) & $p$-Value \\
\hline Operative time (min)* & $280 \pm 43$ & $442 \pm 133$ & $<0.05$ \\
Blood loss (ml)* & $93 \pm 82$ & $864 \pm 107$ & $<0.05$ \\
Resection stump diagnosis (n) & & & NS \\
No atypical cells & 1 & 6 & \\
Low-grade dysplasia & 3 & 0 & \\
Intermediate-grade dysplasia & 0 & 1 & \\
High-grade dysplasia & 0 & 0 & \\
Final pathological diagnosis (n) & & & NS \\
Low-grade dysplasia & 3 & 4 & \\
Intermediate-grade dysplasia & 1 & 2 & \\
High-grade dysplasia & 0 & 1 & \\
IPMN subtype (n) & & & \\
Gastric & 2 & 4 & \\
Intestinal & 2 & 1 & \\
Gastric/intestinal & 0 & 0 & \\
NA & 0 & 2 & \\
Complications n (\%) & $1(25 \%)$ & $3(43 \%)$ & NS \\
Grade B PF & $0(0 \%)$ & $1(14 \%)$ & \\
DGE & $1(25 \%)$ & $2(29 \%)$ & \\
Clavien IIIa or more & $1(25 \%)$ & $3(43 \%)$ & \\
Re-laparotomy & $0(0 \%)$ & $3(43 \%)$ & \\
Length of hospital stay (days)* & $25 \pm 32$ & $36 \pm 18$ & NS \\
De novo or exacerbated DM (n) & 0 & 3 & NS \\
Recurrence (n) & 0 & 0 & NS \\
\hline & & & \\
\hline
\end{tabular}

* Mean \pm standard deviation. IPMN: Intraductal papillary mucinous neoplasm; EN: enucleation; PD: pancreaticoduodenectomy; POPF: postoperative pancreatic fistula; DGE: delayed gastric emptying; DM: diabetes mellitus; NA: not applicable; NS: not significant, $p \geq 0.05$.

Furthermore, we did not experience grade B pancreatic fistula in the enucleation group; these results are better than those of previous work, which reported a pancreatic fistula rate of $0 \%$ to $43 \%$ (10-12). The operative courses were very smooth without complications in the last three consecutive patients except for the first patient, who developed functional duodenal stenosis lasting for 2 months; however, this resolved with conservative treatment. The precise reasons for this complication are unknown, but stenosis of the third portion of the duodenum might have occurred due to vascular impairment and fluid collection around the dissected area. After experiencing this complication, we made every effort to preserve as many vessels as possible along the third portion of the duodenum (e.g. anterior inferior pancreaticoduodenal artery and vein) during dissection of the UP and decided not to perform Kocher's maneuver.

The most important technical tip for successful enucleation is meticulous and accurate dissection of the plane between the pancreatic parenchyma and the thin tumor wall with the use of a mosquito dissector. This dissection is usually smooth, without injury to the pancreatic parenchyma. 

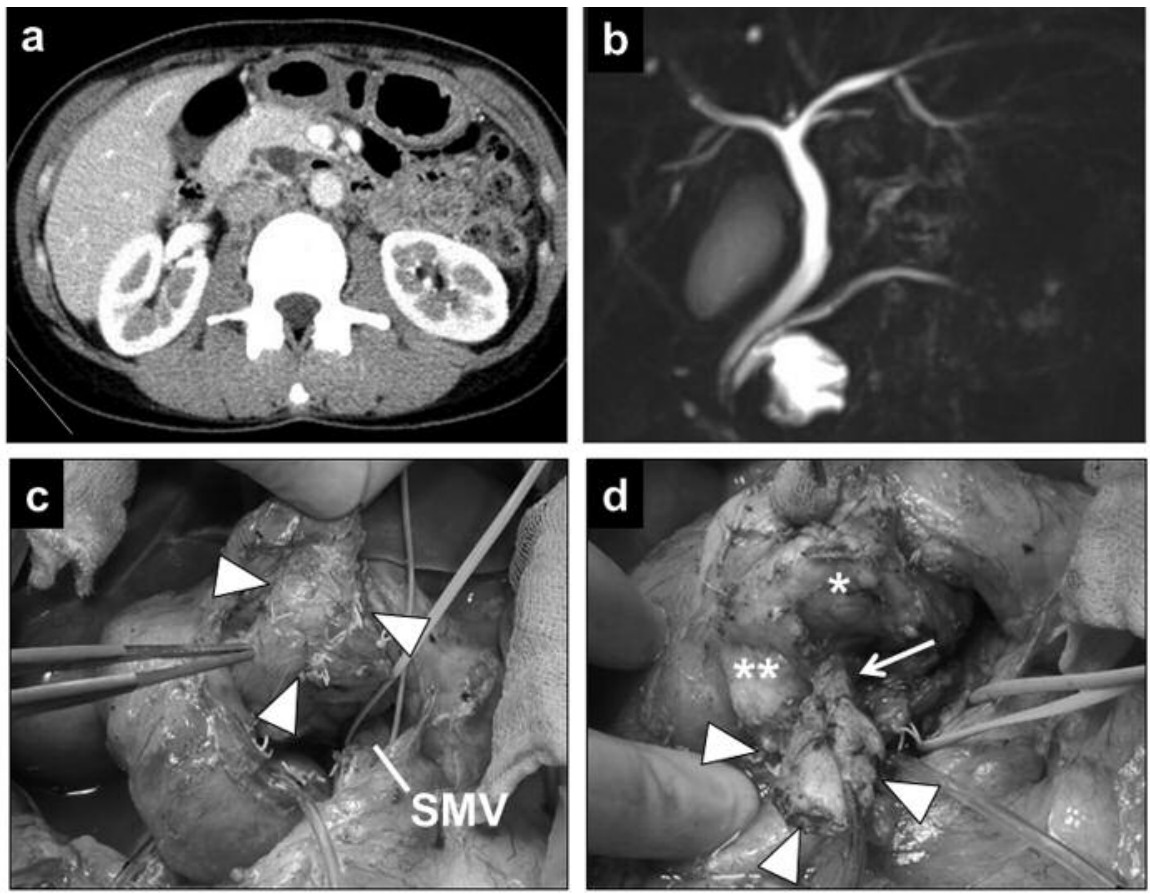

Figure 1. a: Preoperative computed tomographic scan of a patient in the enucleation group. Note that a cystic tumor (24 mm) is located in the uncinate process of the pancreas, while the main pancreatic duct is not dilated. No mural nodules are evident. $b$ : Magnetic resonance cholangiopancreatography of the same patient. c: Operative view after dissection and mobilization of the uncinate process from the superior mesenteric vein (blue tape) and the third portion of the duodenum. Note that the wall of the intraductal papillary mucinous neoplasm (arrowheads) can be identified from the surface. SMV, Superior mesenteric vein. d: The intraductal papillary mucinous neoplasm was present between the dorsal (asterisk) and ventral (double asterisks) pancreas. Completion of the dissection of the intraductal papillary mucinous neoplasm revealed the connection of the intraductal papillary mucinous neoplasm to the main pancreatic duct by its stalk (arrow).

When the adhesion between the cyst wall and parenchyma is too difficult to dissect, a portion of the pancreatic parenchyma should be ligated and divided to avoid injuring the IPMN wall. Spillage of the tumor content can be prevented by using this technique in an accurate plane. We should be very cautious to avoid spillage of the IPMN content because development of pseudomyxoma peritonei after such spillage has been reported (22). Furthermore, no pancreatic fistulas developed in the enucleation group, which contrasts sharply with previous reports describing a pancreatic fistula rate of $30 \%$ to $45 \%$ (10-12). Moreover, safe preservation of the main pancreatic duct is crucial for this procedure. To avoid injury to the main pancreatic duct during enucleation, we routinely place the ENPD tube 2 to 3 days before surgery and perform repeated intraoperative pancreatography and a leakage test with indocyanine green solution. However, we believe that this procedure can be omitted with cumulative experience and a better understanding of the anatomy involved. Laparoscopic enucleation for BD-IPMN has been reported sporadically (23). However, we believe that laparoscopic enucleation of
BD-IPMN is very difficult and dangerous because delicate and meticulous dissection is not possible with currently available instruments, increasing the risk of spillage of IPMN content.

From an oncological point of view, controversy exists regarding the indications for less invasive pancreatectomy for IPMN. This is probably because preoperative establishment of a definitive diagnosis of benign IPMN is very difficult using the currently available diagnostic modalities (24). Nonetheless, preoperative diagnostic procedures to exclude malignant lesions are of paramount importance in the performance of enucleation. Therefore, we routinely perform full preoperative workups for enucleation candidates, including computed tomography, magnetic resonance cholangiopancreatography, positron-emission tomography, EUS, and multiple pancreatic juice cytology examinations after ERPD tube placement.

A possible drawback of enucleation from an oncological viewpoint is that no long-term recurrence or survival rates are yet available. Furthermore, progression of BD-IPMN to the main pancreatic duct cannot be accurately assessed 
preoperatively as well as postoperatively (25). As revealed in this study, the presence of low-grade dysplasia on the stump of the enucleated BD-IPMN was a frequent and unavoidable finding. However, several authors have reported that low-grade dysplasia on the resection stump did not affect the long-term results (26). Based on these findings, the 2012 consensus guidelines allowed remnant low-grade dysplasia on the resection stump. Therefore, we currently believe that the presence of low-grade dysplasia on the resection stump after enucleation is acceptable and that further resection is unnecessary. If a frozen section of the stump reveals a malignant lesion, standard pancreatectomy with lymphadenectomy should be performed. As such, we think that enucleation has a role similar to a total biopsy of BD-IPMN before consecutive or second-look standard pancreatectomy. Accordingly, we believe that enucleation should be indicated for patients with symptomatic BD-IPMN with a low malignant possibility, such as those with negative pancreatic juice cytological findings, normal tumor marker levels, a negative positron-emission tomographic scan, a $\leq 10$-mm mural nodule height (27), and no high-risk stigmata.

In conclusion, enucleation of BD-IPMN at the PH or UP is a feasible and oncologically legitimate procedure in selected patients. However, accumulation of additional cases with a longer follow-up and ideally a prospective randomized study comparing enucleation with standard resection is warranted to confirm the efficacy of this surgical modality.

\section{Conflicts of Interest}

The Authors have no conflicts of interest in regard to this study.

\section{References}

1 Tanaka M: Current roles of endoscopy in the management of intraductal papillary mucinous neoplasm of the pancreas. Dig Endosc 27: 450-457, 2015.

2 Marchegiani G, Mino-Kenudson M, Sahora K, MoralesOyarvide V, Thayer S, Ferrone C, Warshaw AL, Lillemoe KD and Fernández-Del Castillo C: IPMN involving the main pancreatic duct: biology, epidemiology, and long-term outcomes following resection. Ann Surg 261: 976-983, 2015.

3 Daudé M, Muscari F, Buscail C, Carrère N, Otal P, Selves J, Buscail L and Bournet B: World J Gastroenterol 21: 2658-2667, 2015.

4 Kim YI, Shin SH, Song KB, Hwang DW, Lee JH, Park KM, Lee YJ and Kim SC: Branch duct intraductal papillary mucinous neoplasm of the pancreas: single-center experience with 324 patients who underwent surgical resection. Korean J Hepatobiliary Pancreat Surg 19: 113-120, 2015.

5 Topal B, Fieuws S, Aerts R, Weerts J, Feryn T, Roeyen G, Bertrand C, Hubert C, Janssens M and Closset J: Pancreaticojejunostomy versus pancreaticogastrostomy reconstruction after pancreaticoduodenectomy for pancreatic or periampullary tumours: a multicentre randomised trial. Lancet Oncol 14: 655662,2013
6 Rault A, SaCunha A, Klopfenstein D, Larroudé D, Epoy FN, Collet D and Masson B: Pancreaticojejunal anastomosis is preferable to pancreaticogastrostomy after pancreaticoduodenectomy for longterm outcomes of pancreatic exocrine function. J Am Coll Surg 201: 239-244, 2005.

7 Hirono S, Murakami Y, Tani M, Kawai M, Okada K, Uemura K, Sudo T, Hashimoto Y, Nakagawa N, Kondo N and Yamaue H: Identification of risk factors for pancreatic exocrine insufficiency after pancreaticoduodenectomy using a 13C-labeled mixed triglyceride breath test. World J Surg 39: 516-525, 2015.

8 Song KB, Kim SC, Hwang DW, Lee JH, Lee DJ, Lee JW, Jun ES, Sin SH, Kim HE, Park KM and Lee YJ: Enucleation for benign or low-grade malignant lesions of the pancreas: Singlecenter experience with 65 consecutive patients. Surgery 158 : 1203-1210, 2015.

9 Faitot F, Gaujoux S, Barbier L, Novaes M, Dokmak S, Aussilhou B, Couvelard A, Rebours V, Ruszniewski P, Belghiti $\mathrm{J}$ and Sauvanet A: Reappraisal of pancreatic enucleations: A single-center experience of 126 procedures. Surgery 158: 201$210,2015$.

10 Sauvanet A, Gaujoux S, Blanc B, Couvelard A, Dokmak S, Vullierme MP, Ruszniewski $P$, Belghiti $J$ and Lévy P: Parenchyma-sparing pancreatectomy for presumed noninvasive intraductal papillary mucinous neoplasms of the pancreas. Ann Surg 260: 364-371, 2014.

11 Hwang HK, Park JS, Kim JK, Park CM, Cho SI and Yoon DS: Comparison of efficacy of enucleation and pancreaticoduodenectomy for small $(<3 \mathrm{~cm})$ branch duct type intraductal papillary mucinous neoplasm located at the head of pancreas and the uncinate process. Yonsei Med J 53: 106-110, 2012.

12 Turrini O, Schmidt CM, Pitt HA, Guiramand J, AguilarSaavedra JR, Aboudi S, Lillemoe KD and Delpero JR: Sidebranch intraductal papillary mucinous neoplasms of the pancreatic head/uncinate: resection or enucleation? HPB 13: 126-131, 2011.

13 Nishizaki T, Ijichi H, Terashi T, Shimabukuro R, Maruyama S, Guntani A, Yamaoka T, Inoue H, Shiraishi T, Wada H and Takahashi I: Pancreatogastrostomy with an elastic purse-string suture around the gastric orifice after pancreatoduodenectomy. Surg Today 39: 548-551, 2009.

14 Bassi C, Dervenis C, Butturini G, Fingerhut A, Yeo C, Izbicki J, Neoptolemos J, Sarr M, Traverso W, Buchler M; International Study Group on Pancreatic Fistula Definition. Postoperative pancreatic fistula: an international study group (ISGPF) definition. Surgery 138: 8-13, 2005.

15 Dindo D, Demartines N and Clavien PA: Classification of surgical complications: a new proposal with evaluation in a cohort of 6336 patients and results of a survey. Ann Surg 240: 205-213, 2004.

16 Tanaka M, Fernández-del Castillo C, Adsay V, Chari S, Falconi M, Jang JY, Kimura W, Levy P, Pitman MB, Schmidt CM, Shimizu M, Wolfgang CL, Yamaguchi K, Yamao K; International Association of Pancreatology. International consensus guidelines 2012 for the management of IPMN and MCN of the pancreas. Pancreatology 12: 183-197, 2012.

17 Goh BK, Lin Z, Tan DM, Thng CH, Khor CJ, Lim TK, Ooi LL and Chung AY: Evaluation of the Fukuoka Consensus Guidelines for intraductal papillary mucinous neoplasms of the pancreas: Results from a systematic review of 1,382 surgically resected patients. Surgery 158: 1192-1202, 2015. 
18 Sahora K, Mino-Kenudson M, Brugge W, Thayer SP, Ferrone CR, Sahani D, Pitman MB, Warshaw AL, Lillemoe KD and Fernandez-del Castillo CF: Branch duct intraductal papillary mucinous neoplasms: does cyst size change the tip of the scale? A critical analysis of the revised international consensus guidelines in a large single-institutional series. Ann Surg 258: 466-475, 2013.

19 Nakagohri T, Kinoshita T, Konishi M, Takahashi S, Gotohda N, Kobayashi S, Kojima M, Miyauchi H and Asano T: Inferior head resection of the pancreas for intraductal papillary mucinous neoplasms. J Hepatobiliary Pancreat Sci 17: 798-802, 2010.

20 Beger HG, Rau BM, Gansauge F, Schwarz M, Siech M and Poch B: Duodenum-preserving total pancreatic head resection for cystic neoplasm: a limited but cancer-preventive procedure. Langenbecks Arch Surg 393: 589-598, 2008.

21 Tsuchikawa T, Hirano S, Tanaka E, Kato K, Matsumoto J, Nakamura T, Ebihara $\mathrm{Y}$, Shichinohe $\mathrm{T}$ and Miyamoto $\mathrm{M}$ : Modified duodenum-preserving pancreas head resection for lowgrade malignant lesion in the pancreatic head. Pancreatology 13: 170-174, 2013.

22 Mizuta Y, Akazawa Y, Shiozawa K, Ohara H, Ohba K, Ohnita $\mathrm{K}$, Isomoto H, Takeshima F, Omagari K, Tanaka K, Yasutake T, Nakagoe T, Shirono K and Kohno S: Pseudomyxoma peritonei accompanied by intraductal papillary mucinous neoplasm of the pancreas. Pancreatology 5: 470-474, 2005.

23 Thomas E, Matsuoka L, Alexopoulos S, Selby R and Parekh D: Laparoscopic hand-assisted parenchymal-sparing resections for presumed side-branch intraductal papillary mucinous neoplasms. J Laparoendosc Adv Surg Tech A 25: 668-671, 2015.
24 Fritz S and Lerch MM: Natural history and management of intraductal papillary mucinous neoplasms: Current Evidence. Viszeralmedizin 31: 25-30, 2015.

25 Fritz S, Klauss M, Bergmann F, Strobel O, Schneider L, Werner $\mathrm{J}$, Hackert $\mathrm{T}$ and Büchler MW: Pancreatic main-duct involvement in branch-duct IPMNs: an underestimated risk. Ann Surg 260: 848-855, 2014; discussion 855-856.

26 D'Angelica M, Brennan MF, Suriawinata AA, Klimstra D and Conlon KC: Intraductal papillary mucinous neoplasms of the pancreas: an analysis of clinicopathologic features and outcome. Ann Surg 239: 400-408, 2004.

27 Kobayashi G, Fujita N, Maguchi H, Tanno S, Mizuno N, Hanada K, Hatori T, Sadakari Y, Yamaguchi T, Tobita K, Doi R, Yanagisawa A, Tanaka M; Working Group for the Natural History of IPMN of the Japan Pancreas Society. Natural history of branch duct intraductal papillary mucinous neoplasm with mural nodules: a Japan Pancreas Society multicenter study. Pancreas 43: 532-538, 2014.
Received October 28, 2016

Revised November 14, 2016

Accepted November 15, 2016 